\title{
UNIQUENESS OF THE OPTIMAL CONTROL FOR A LOTKA-VOLTERRA CONTROL PROBLEM WITH A LARGE CROWDING EFFECT
}

\author{
J.L. GÁMEZ AND J.A. MONTERO
}

\begin{abstract}
Uniqueness of the optimal control is obtained by assuming certain conditions on the crowding effect of the species. Moreover, an approximation procedure for the unique optimal control is developed.
\end{abstract}

\section{INTRODUCTION}

In this work we study an optimal control problem whose steady-state equation is a P.D.E. of Lotka-Volterra type:

$$
\begin{array}{cl}
-\Delta u(x)=u(x)[a(x)-f(x)-b(x) u(x)], & x \in \Omega, \\
u(x)=0, & x \in \partial \Omega,
\end{array}
$$

where $\Omega$ is a bounded and regular domain in $\mathbb{R}^{N}$. The unknown $u$ represents the concentration of the biological species. The coefficients which appear in (1.1) can be interpreted as follows: function $a$ is the growth rate, function $b$ means the crowding effect and $f$ denotes a control on the process of production (see [14]). The space of admissible controls is

$$
L_{+}^{\infty}(\Omega)=\left\{g \in L^{\infty}(\Omega): g(x) \geq 0 \text { a.e. in } \Omega\right\} .
$$

Under the hypothesis

$$
[\mathbf{H}]: a, b \in L^{\infty}(\Omega), \operatorname{essinf}_{\Omega} b>0,
$$

the equation (1.1) has, for every admissible control $f$, a unique maximal nonnegative weak solution which will be denoted by $u_{\Omega, a, b, f}$ (see [2]).

The benefit-payoff functional may be given by

$$
J_{K, L}(f)=\int_{\Omega}\left[K u_{\Omega, a, b, f}(x) f(x)-L f^{2}(x)\right] d x,
$$

which express the difference between profit and cost. Here, $K>0$ denotes the sale price of the species, and $L>0$ is the cost of the control. To study the existence of maximum for $J_{K, L}$ and some additional properties, we prefer to consider the re-scaled functional $J=\frac{J_{K, L}}{L}$, this is

$$
J(f)=\int_{\Omega}\left(\lambda u_{\Omega, a, b, f}(x) f(x)-f^{2}(x)\right) d x,
$$

Dep. Análisis Matemático, Universidad de Granada. 18071-Granada, Spain. E-mail: jlgamez@goliat.ugr.es - jmontero@goliat.ugr.es.

Received by the journal May 28, 1996. Accepted for publication November 8, 1996.

The authors have been supported in part by the "Dirección General de Enseñanza Superior, Ministerio de Educación y Ciencia" (Spain), under grant number PB95-1190 and by EEC contract, Human Capital and Mobility program, ERBCHRXCT940494.

(C) Société de Mathématiques Appliquées et Industrielles. Typeset by LATEX. 
where the constant $\lambda>0$ denotes the rate between the sale price of the biological species and the cost of the control.

We denote by $\left(P_{\Omega, a, b, \lambda}\right)$ the problem of finding an admissible control, $f$, such that

$$
J(f)=\sup _{g \in L_{+}^{\infty}(\Omega)} J(g) .
$$

Such a control, $f$, will be called an "optimal control" of problem $\left(P_{\Omega, a, b, \lambda}\right)$.

The existence of optimal controls under hypothesis [H] has been proved in [4], where a necessary and sufficient condition to obtain the positivity of the optimal benefit is given.

We are interested in finding conditions which guarantee the uniqueness of the optimal control and its approximation.

In [5] it was obtained the uniqueness and an approach scheme for the optimal control was developed, in the case where $\Omega, a, b$ are fixed, and $\lambda>0$ is small enough. The result can also be applied for $\Omega, a, \lambda$ fixed, and $b$ a sufficiently large positive constant, or $b=\gamma b_{0}$, where $\gamma \in \mathbb{R}$ is a a sufficiently large constant and function $b_{0}$ satisfies $[\mathbf{H}]$. However, the case where $b$ is a nonconstant arbitrary function does not seem to be approachable by that method.

This last case is discussed here. We fix $\Omega, \lambda$ and $a$, and we prove uniqueness of the optimal control, provided that ess sup $b \leq$ Messinf $b$, for some $1 \leq M<2$, and essinf $b$ is large enough (see Theorem 3.5).

Similar problems are studied in $[8,10,11,13]$. In [11] the authors have studied a control problem with a steady-state equation similar to (1.1), but with Neumann boundary conditions. Their space of admissible controls is

$$
C_{\delta}=\left\{g \in L^{\infty}(\Omega): 0 \leq g(x) \leq \delta \text { a.e. in } \Omega\right\},
$$

where $0<\delta<\operatorname{essinf}_{\Omega} a(x)$. In this particular situation they prove the existence of an optimal control $f \in C_{\delta}$. Under suitable conditions, they describe the optimal control $f$ in terms of the solution of an appropriate elliptic system (the optimality system). In [8] the periodic problem for the parabolic model is considered.

In section 2 we fix some notation, and we also recall some results obtained in $[4,5]$. In section 3 we obtain the optimality system, which is used to prove the uniqueness of the optimal control. In section 4 we provide an iterative scheme to approximate the unique solution of the optimality system.

\section{Preliminary Results}

For a bounded function $e \in L_{+}^{\infty}(\Omega)$, we denote $\bar{e}=\operatorname{ess} \sup _{\Omega} e$, and accordingly $\underline{\epsilon}=\operatorname{ess} \inf _{\Omega} \epsilon$. From now on we consider the equation (1.1), assuming the hypothesis $[\mathbf{H}]$.

For every $q \in L^{\infty}(\Omega)$, we define $\sigma_{1}(q)$ to be the principal eigenvalue of the following eigenvalue problem:

$$
\begin{array}{cc}
-\Delta u(x)+q(x) u(x)=\sigma u(x), & x \in \Omega, \\
u(x)=0, & x \in \partial \Omega .
\end{array}
$$

Esaim: Cocv, January 1997 , Vol.2, Pp. 1-12 
It is known (see for instance [6]) that $\sigma_{1}(q)$ satisfies the variational characterization

$$
\sigma_{1}(q)=\inf _{u \in H_{0}^{1}(\Omega) \backslash\{0\}} \frac{\int_{\Omega}|\nabla u|^{2}+\int_{\Omega} q u^{2}}{\int_{\Omega} u^{2}}
$$

where $H_{0}^{1}(\Omega)$ is the usual Sobolev space. It is also known that $\sigma_{1}(q)$ has algebraic multiplicity equal to one. Moreover, one can choose an associated eigenfunction, $\phi_{1}(q)$, such that $\phi_{1}(q) \in C^{1, \alpha}(\bar{\Omega})$ (the space of Hölderian functions), $\forall \alpha \in(0,1), \phi_{1}(q)$ strictly positive in $\Omega$, and $\left\|\phi_{1}(q)\right\|_{L^{\infty}(\Omega)}=1$.

As a consequence of $(2.1)$, it follows that $\sigma_{1}(q)$ has the following properties:

i) If $q_{1}, q_{2} \in L^{\infty}(\Omega)$, with $q_{1}(x) \leq q_{2}(x)$, a.e. in $\Omega$, then $\sigma_{1}\left(q_{1}\right) \leq \sigma_{1}\left(q_{2}\right)$. Moreover, if the set $\left\{x \in \Omega: q_{1}(x)<q_{2}(x)\right\}$ has positive measure, then $\sigma_{1}\left(q_{1}\right)<\sigma_{1}\left(q_{2}\right)$.

ii) $\forall q \in L^{\infty}(\Omega), \forall M \in \mathbb{R}, \sigma_{1}(q+M)=\sigma_{1}(q)+M$.

iii) $\sigma_{1}(q)$ depends continuously on $q \in L^{\infty}(\Omega)$.

We now recall some properties of the Schrödinger operator $(-\Delta+q)$.

Lemma 2.1. Consider $q \in L^{\infty}(\Omega)$, satisfying $\sigma_{1}(q)>0$. Then:

i) For each $f \in L^{2}(\Omega)$, the linear problem

$$
\begin{array}{cc}
-\Delta u+q u=f, & \text { in } \Omega, \\
u=0, & \text { on } \partial \Omega,
\end{array}
$$

admits a unique weak solution $u \in H_{0}^{1}(\Omega)$. Moreover, if $f \in L^{\infty}(\Omega)$, then $u \in C^{1, \alpha}(\bar{\Omega}), \forall \alpha \in(0,1)$.

ii) Let $u_{1}, u_{2} \in H^{1}(\Omega)$ be such that $\forall \phi \in H_{0}^{1}(\Omega), \phi \geq 0$,

$$
\begin{gathered}
\int_{\Omega} \nabla u_{1} \nabla \phi+\int_{\Omega} q u_{1} \phi \leq \int_{\Omega} \nabla u_{2} \nabla \phi+\int_{\Omega} q u_{2} \phi, \\
u_{1} \leq u_{2} \text { on } \partial \Omega .
\end{gathered}
$$

Then $u_{1} \leq u_{2}$ in $\Omega$. We remark here that the inequality $u_{1} \leq u_{2}$ on $\partial \Omega$ means $\left(u_{1}-u_{2}\right)^{+} \in H_{0}^{1}(\Omega)$.

iii) Consider $f \in L^{2}(\Omega), f(x) \geq 0$ a.e. in $\Omega$, and $p_{1}, p_{2} \in L^{\infty}(\Omega)$, with $p_{1}(x) \geq p_{2}(x)$ a.e. in $\Omega$, and $\sigma_{1}\left(p_{2}\right)>0$. We denote by $\omega_{i}(i=1,2)$, the unique weak solution (it exists from $i$ )) of problem

$$
\begin{array}{cc}
-\Delta \omega_{i}+p_{i} \omega_{i}=f, & \text { in } \Omega, \\
\omega_{i}=0, & \text { on } \partial \Omega .
\end{array}
$$

Then

$$
\omega_{1}(x) \leq \omega_{2}(x) \text { a.e. in } \Omega \text {. }
$$

Proof. Part $i$ ) can be found in [5]. Parts ii) and iii) are proved in [12].

We also point out some known results about problem (1.1):

Lemma 2.2. (see $[2,3,7]$ ) Assume hypothesis $[\mathbf{H}]$. Then, problem (1.1) has a nonnegative and nontrivial weak solution if and only if $\sigma_{1}(-a+f)<0$. Moreover, in this case the nonnegative and nontrivial solution is unique. So, for any $f \in L^{\infty}(\Omega)$, we can define $u_{\Omega, a, b, f}$ to be the maximal nonnegative 
solution of equation (1.1). For $\sigma_{1}(-a+f)<0$ one has $u_{\Omega, a, b, f} \in C^{1}(\bar{\Omega})$, and the following a priori bounds:

$$
\frac{-\sigma_{1}(-a+f)}{\bar{b}} \phi_{1}(-a+f)(x) \leq u_{\Omega, a, b, f}(x) \leq \frac{\bar{a}-\underline{f}}{\underline{b}}, \forall x \in \Omega .
$$

Consequently, $u_{\Omega, a, b, f} \equiv 0$ if $\sigma_{1}(-a+f) \geq 0$ and $u_{\Omega, a, b, f}$ is strictly positive in $\Omega$ if $\sigma_{1}(-a+f)<0$. Moreover, for $\Omega, a, b$ fixed, the map $L^{\infty}(\Omega) \rightarrow C^{1}(\bar{\Omega})$, $f \mapsto u_{\Omega, a, b, f}$ is continuous.

Taking into account that every sufficiently large constant is an uppersolution of (1.1) (we refer to Amann [1] for the definition of lower- and upper-solutions), one infers that every bounded lower-solution, $w$, of (1.1) must satisfy

$$
w(x) \leq u_{\Omega, a, b, f}(x), \forall x \in \Omega .
$$

We now recall some of the results obtained in $[4,5]$.

Theorem 2.3. ([4], Existence of optimal control). Assume hypothesis $[\mathbf{H}]$. Then there exists an optimal control for problem $\left(P_{\Omega, a, b, f}\right)$, i.e.

$$
\exists f \in L_{+}^{\infty}(\Omega) \text { such that } J(f)=\sup _{g \in L_{+}^{\infty}(\Omega)} J(g) .
$$

Moreover if $\bar{a} \leq 0$ then $f \equiv 0$, and if $\bar{a}>0$, the optimal control $f$ satisfies

$$
f \leq \lambda \frac{\bar{a}}{\underline{b}} \text { a.e. in } \Omega \text {. }
$$

Theorem 2.4. ([4], Positivity of benefit). Assume hypothesis [H]. Then,

$$
\sup _{g \in L_{+}^{\infty}(\Omega)} J(g)>0 \Longleftrightarrow \sigma_{1}(-a)<0 .
$$

REMARK 2.5. 1. Observe that we have not imposed any upper bound on the control space, but relation (2.3) implies that the space of "interesting" controls is (in fact) bounded.

2. Theorem 2.3 justifies the hypothesis $\sigma_{1}(-a)<0$ in many of the next results. In particular, observe that $\sigma_{1}(-a)<0 \Rightarrow \bar{a}>0$.

Lemma 2.6. ([5, Lemma 3.2]). Assume $[\mathbf{H}]$ and $\sigma_{1}(-a)<0$. If $f \in L_{+}^{\infty}(\Omega)$ is an optimal control, then

$$
f=\frac{\lambda}{2} u_{\Omega, a, b, f}\left(1-p_{\Omega, a, b, f}\right)^{+}, \text {a.e. in } \Omega,
$$

where $p_{\Omega, a, b, f}$ is the unique solution of the linear problem

$$
\begin{array}{cc}
-\Delta p_{\Omega, a, b, f}+\left(-a+f+2 b u_{\Omega, a, b, f}\right) p_{\Omega, a, b, f}=f, & \text { in } \Omega, \\
p_{\Omega, a, b, f}=0 & \text { on } \partial \Omega
\end{array}
$$

(Observe that $\left.\sigma_{1}\left(-a+f+2 b u_{\Omega, a, b, f}\right)>\sigma_{1}\left(-a+f+b u_{\Omega, a, b, f}\right)=0\right)$.

\section{Optimality System AND UNIQUENESS OF THE OPTIMAL CONTROL}

In this section, under suitable conditions involving function $b$, we are going to prove that any optimal control can be expressed in terms of a solution of a certain system, the optimality system (3.8)-(3.9).

We provide conditions to assure the uniqueness of solution for the optimality system and therefore, uniqueness of optimal control. 
We will fix $\Omega, a$ and $\lambda$, and we will look for conditions on function $b$ to obtain the uniqueness of the optimal control. For the sake of simplicity, from now on, we denote by $u_{b, f}$ the maximal nonnegative solution of (1.1), instead of $u_{\Omega, a, b, f}$, and by $p_{b, f}$ the solution of (2.4), instead of $p_{\Omega, a, b, f}$.

Our first objective is to find conditions on function $b$ to guarantee, for any optimal control $f \in L_{+}^{\infty}(\Omega)$, the following estimation on the solution of $(2.4)$ :

$$
p_{b, f} \leq 1 \text {, a.e. in } \Omega \text {. }
$$

In this case, we will be able to express any optimal control in terms of the solution of an appropriate system of P.D.E's (see Theorem 3.3 below).

To obtain this estimate we will use the statements ii)-iii) of Lemma 2.1 . In fact, as a consequence of $i i)$ we deduce that $p_{b, f} \geq 0$ in $\Omega$. To get an upper bound for $p_{b, f}$, it will be sufficient to find an upper bound for $f$ and a lower bound for the expression $-a+f+2 b u_{b, f}$.

Lemma 3.1. Consider $a, b$ satisfying $[\mathbf{H}], \sigma_{1}(-a)<0$ and

$$
\bar{b} \leq M \underline{b}, \text { for some } 1 \leq M<2 .
$$

One may choose $\varepsilon \in \mathbb{R}^{+}$such that

$$
\varepsilon<\frac{M}{2} \sigma_{1}\left(-a+\frac{2}{M} u_{1,0}\right) .
$$

Then, there exists a positive constant $b_{0}$ (which depends on $\Omega, a, \lambda$ and $\varepsilon$ ), such that if $\underline{b} \geq b_{0}$ and $f$ is any optimal control, the function $p_{b, f}$ defined in (2.4) satisfies the estimates

$$
0 \leq p_{b, f} \leq \frac{Q}{\underline{b}}, \text { a.e. in } \Omega,
$$

where $Q$ is the unique solution of

$$
\begin{array}{cc}
-\Delta Q+\left(-a+\frac{2}{M}\left(u_{1,0}-\varepsilon\right)\right) Q=\lambda \bar{a}, & \text { in } \Omega, \\
Q=0, & \text { on } \partial \Omega .
\end{array}
$$

Proof. As a consequence of the hypothesis $\sigma_{1}(-a)<0$ the function $u_{1,0}$ is strictly positive in $\Omega$ and therefore $\sigma_{1}\left(-a+u_{1,0}\right)=0$. So,

$$
\sigma_{1}\left(-a+\frac{2}{M} u_{1,0}\right)>0 .
$$

Due to the choice of $\varepsilon$ and the statement $i)$ of Lemma 2.1, there exists a unique solution $Q$ of (3.3). From the continuity of the map $L^{\infty}(\Omega) \rightarrow C^{1}(\bar{\Omega})$, $f \mapsto u_{1, f}$ (see Lemma 2.2), there exists a positive constant $b_{0}$ such that for $\underline{b} \geq b_{0}$, if $g \in L_{+}^{\infty}(\Omega)$ with $g \leq \lambda \frac{\bar{a}}{\underline{b}}$, then

$$
u_{1, g} \geq u_{1,0}-\varepsilon \text { a.e. in } \Omega \text {. }
$$

If $f$ is an optimal control for $\left(P_{\Omega, a, b, \lambda}\right)$, then by using that $u_{\bar{b}, f}$ is a lowersolution of (1.1) and that $k u_{k, f}=u_{1, f} \forall k>0$, it follows

$$
2 b u_{b, f}=2 \frac{b}{\bar{b}} \bar{b} u_{b, f} \geq \frac{2}{M} \bar{b} u_{\bar{b}, f}=\frac{2}{M} u_{1, f} .
$$

Moreover since $\underline{b} \geq b_{0}$, from $(2.3)$ and (3.4) we obtain

$$
-a+f+2 b u_{b, f} \geq-a+2 b u_{b, f} \geq-a+\frac{2}{M}\left(u_{1,0}-\varepsilon\right) \text { a.e. in } \Omega \text {. }
$$


The function $\frac{Q}{\underline{b}}$ satisfies

$$
-\Delta\left(\frac{Q}{\underline{b}}\right)+\left(-a+\frac{2}{M}\left(u_{1,0}-\varepsilon\right)\right) \frac{Q}{\underline{b}}=\lambda \frac{\bar{a}}{\underline{b}} .
$$

From ii) and iii) of Lemma 2.1 taking into account that $\lambda \frac{\bar{a}}{\underline{b}} \geq f$ and the inequality (3.6), we conclude the proof of lemma 3.1 .

Corollary 3.2. Assume $[\mathbf{H}], \sigma_{1}(-a)<0$, (3.1) and

$$
\underline{b} \geq b_{1} \equiv \max \left\{b_{0},\|Q\|_{\infty}\right\} .
$$

Then for any optimal control $f$, the function $p_{b, f}$ defined in (2.4) satisfies:

$$
0 \leq p_{b, f} \leq 1 \text {, a.e. in } \Omega \text {. }
$$

Theorem 3.3. (Optimality system). Let us assume $[\mathbf{H}], \sigma_{1}(-a)<0,(3.1)$ and (3.7). Then, any optimal control $f \in L_{+}^{\infty}(\Omega)$, can be expressed as

$$
f=\frac{\lambda}{2} u_{b, f}\left(1-p_{b, f}\right)
$$

where the pair $(u, p) \equiv\left(u_{b, f}, p_{b, f}\right)$, is a solution of the optimality system:

$$
\begin{gathered}
-\Delta u=u\left(a-\left[b+\frac{\lambda}{2}(1-p)\right] u\right), \text { in } \Omega, \\
-\Delta p+p(-a+2 b u)=\frac{\lambda}{2} u(1-p)^{2}, \text { in } \Omega, \\
u=p=0, \text { on } \partial \Omega,
\end{gathered}
$$

satisfying

$$
0 \leq p \leq 1, u>0 \text {, a.e. in } \Omega \text {. }
$$

Proof. It is a direct consequence of previous results.

Lemma 3.4. Let us assume $[\mathbf{H}], \sigma_{1}(-a)<0$, (3.1) and (3.7). Then any solution $(v, q)$ of system (3.8) under the conditions (3.9) satisfies

$$
w(x) \leq v(x) \leq \frac{\bar{a}}{\underline{b}}, \quad 0 \leq q(x) \leq \frac{Q(x)}{\underline{b}}, \text { a.e. in } \Omega,
$$

where $w$ is the unique maximal nonnegative solution of

$$
\begin{array}{cl}
-\Delta w=w\left[a-\frac{\lambda}{2} w-b w\right] & \text { in } \Omega, \\
w=0 & \text { on } \partial \Omega,
\end{array}
$$

and $Q$ is defined by (3.3).

Moreover, by choosing $\varepsilon$ as in (3.2), one has

$$
2 b(x) w(x) \geq \frac{2}{M}\left(u_{1,0}(x)-\varepsilon\right) \text { a.e. in } \Omega .
$$

Proof. Observe that $v$ is a nonnegative and nontrivial solution of an equation similar to (1.1), so, estimates of type (2.2) hold, obtaining $0<v(x) \leq \frac{\bar{a}}{\underline{b}}$ a.e. in $\Omega$. The function $w$ is a bounded lower-solution for the equation satisfied by $v$, therefore

$$
w(x) \leq v(x) \leq \frac{\bar{a}}{\underline{b}} \text { a.e. in } \Omega .
$$


By using the established notation for the solutions of (1.1), we have $w=$ $u_{b, \frac{\lambda}{2} w}$. Then, similarly to (3.5) and (3.4),

$$
2 b w=2 b u_{b, \frac{\lambda}{2} w} \geq \frac{2}{M} \bar{b} u_{\bar{b}, \frac{\lambda}{2} w}=\frac{2}{M} u_{1, \frac{\lambda}{2} w} \geq \frac{2}{M}\left(u_{1,0}-\varepsilon\right) \text { a.e. in } \Omega .
$$

To prove $q \leq \frac{Q}{\underline{b}}$ in $\Omega$, we denote $h=\frac{\lambda}{2} v(1-q)$. Observe that $q$ satisfies

$$
\begin{array}{cl}
-\Delta q+(-a+h+2 b v) q=h & \text { in } \Omega, \\
q=0 & \text { on } \partial \Omega .
\end{array}
$$

Similarly to the proof of lemma 3.1, by using (3.11), (3.12), the definition of $Q$ and lemma 2.1, the proof is complete.

The main result of this section is the uniqueness of the optimal control. We will use the optimality system and the previous lemma to prove it.

Theorem 3.5. (Uniqueness of the optimal control). Suppose $[\mathbf{H}], \sigma_{1}(-a)<$ 0 and (3.1). Then problem $\left(P_{\Omega, a, b, \lambda}\right)$ admits a unique optimal control, provided that $\underline{b}$ is large enough.

Proof. By choosing $\varepsilon>0$ as in Lemma 3.1, we denote $\delta \equiv \sigma_{1}\left(-a+\frac{2}{M}\left(u_{1,0}-\right.\right.$ $\varepsilon)$ ), which, from (3.2), is strictly positive. Take

$$
\underline{b}^{2} \geq b_{2} \equiv \max \left\{\frac{\lambda \bar{a}^{2}\left\{\frac{\lambda}{2}+2 M\|Q\|_{\infty}\right\}}{2 \delta^{2}}, b_{1}^{2}\right\}
$$

where $Q$ is defined in Lemma 3.1, and $b_{1}$ as in Corollary 3.2.

Fix $\alpha=\frac{1}{\delta}\left(\frac{\lambda}{2}+2 M\|Q\|_{\infty}\right)$. In virtue of Lemma 3.4 , it is easy to check that $(u, p)$ is a solution of system (3.8)-(3.9) if and only if $(u, r) \equiv\left(u, \frac{p}{\alpha}\right)$ is a solution of the following system,

$$
\begin{array}{cl}
-\Delta u-a u+b u^{2}+\frac{\lambda}{2}(1-r \alpha) u^{2}=0, & \text { in } \Omega, \\
-\Delta r+r(-a+2 b u)-\frac{\lambda}{2 \alpha} u(1-r \alpha)^{2}=0, & \text { in } \Omega, \\
u=r=0, & \text { on } \partial \Omega .
\end{array}
$$

with conditions,

$$
0 \leq r(x) \leq \frac{Q(x)}{\alpha \underline{b}}\left(\leq \frac{1}{\alpha}\right), w(x) \leq u(x) \leq \frac{\bar{a}}{\underline{b}}, \text { a.e. in } \Omega .
$$

To show the uniqueness of (3.14), under conditions (3.15). Let us consider another solution $(v, s)$ of $(3.14)-(3.15)$. Then

$$
\begin{aligned}
0= & -\Delta(u-v)-a(u-v)+b\left(u^{2}-v^{2}\right)+ \\
& +\frac{\lambda}{2}\left(u^{2}-v^{2}\right)-\frac{\lambda \alpha}{2}\left(u^{2} r-v^{2} s\right)
\end{aligned}
$$

and

$$
\begin{aligned}
& 0=-\Delta(r-s)-a(r-s)+2 b(u r-v s)- \\
&-\frac{\lambda \alpha}{2}\left(u\left(\frac{1}{\alpha}-r\right)^{2}-v\left(\frac{1}{\alpha}-s\right)^{2}\right) . \\
& \text { Esaim: Cocv, January } 1997 \text {, Vol. } 2, \text { PP. } 1-12
\end{aligned}
$$


Similarly,

$$
\begin{aligned}
0= & -\Delta(u-v)-a(u-v)+b(u-v)(u+v)+ \\
& +\frac{\lambda}{2}(u+v)(u-v)-\frac{\lambda \alpha}{2} u^{2}(r-s)-\frac{\lambda \alpha}{2} s(u+v)(u-v)
\end{aligned}
$$

and

$$
\begin{aligned}
0= & -\Delta(r-s)-a(r-s)+2 b u(r-s)+2 b s(u-v)+ \\
& +\frac{\lambda \alpha}{2} u\left[\frac{2}{\alpha}-(r+s)\right](r-s)-\frac{\lambda \alpha}{2}(u-v)\left(\frac{1}{\alpha}-s\right)^{2} .
\end{aligned}
$$

Multiplying $(3.16)$ by $(u-v),(3.17)$ by $(r-s)$, integrating on $\Omega$, and adding both expressions,

$$
\begin{aligned}
0= & \int_{\Omega}\left[|\nabla(u-v)|^{2}-a(u-v)^{2}+b(u+v)(u-v)^{2}+\right. \\
& \left.+\frac{\lambda}{2}(u+v)(u-v)^{2}(1-\alpha s)-\frac{\lambda \alpha}{2} u^{2}(u-v)(r-s)\right]+ \\
& +\int_{\Omega}\left[|\nabla(r-s)|^{2}-a(r-s)^{2}+2 b u(r-s)^{2}+\right. \\
& +(u-v)(r-s)\left(2 b s-\frac{\lambda \alpha}{2}\left(\frac{1}{\alpha}-s\right)^{2}\right)+ \\
& \left.+\frac{\lambda \alpha}{2} u\left(\frac{2}{\alpha}-(r+s)\right)(r-s)^{2}\right] .
\end{aligned}
$$

Observe that $\frac{\lambda}{2}(u+v)(u-v)^{2}(1-\alpha s)$ and $\frac{\lambda \alpha}{2} u\left(\frac{2}{\alpha}-(r+s)\right)(r-s)^{2}$ are non negative. Moreover, if $r(x) \neq s(x)$ in a subset of $\Omega$ with positive measure then, as a direct consequence of (3.15),

$$
\int_{\Omega} \frac{\lambda \alpha}{2}\left(\frac{2}{\alpha}-(r+s)\right)(r-s)^{2}>0
$$

Now, from (3.15) and (3.12), the functions $(u+v) b$ and $2 b u$ satisfy

$$
\begin{gathered}
(u+v) b \geq \frac{2}{M}\left(u_{1,0}-\varepsilon\right) \\
2 b u \geq \frac{2}{M}\left(u_{1,0}-\varepsilon\right)
\end{gathered}
$$

in $\Omega$.

The variational characterization of $\delta$ (recall (2.1)) implies

$$
\int_{\Omega}|\nabla q|^{2}+\int_{\Omega}\left(-a+\frac{2}{M}\left(u_{1,0}-\varepsilon\right)\right) q^{2} \geq \delta \int_{\Omega} q^{2}, \forall q \in H_{0}^{1}(\Omega) .
$$

Applying this inequality to $q_{1}=u-v$ and $q_{2}=r-s$ and taking into account previous considerations, from (3.18) it follows:

$$
\begin{aligned}
0 \geq & \int_{\Omega}\left[\delta(u-v)^{2}+\delta(r-s)^{2}+\right. \\
& \left.+(u-v)(r-s)\left(2 b s-\frac{\lambda \alpha}{2}\left(\frac{1}{\alpha}-s\right)^{2}-\frac{\lambda \alpha}{2} u^{2}\right)\right] .
\end{aligned}
$$


Moreover, if $r(x) \neq s(x)$ in a subset of $\Omega$ with positive measure then previous inequality is strict. The choice of $\alpha,(3.13)$ and (3.15) imply

$$
\begin{aligned}
\left|2 b s-\frac{\lambda \alpha}{2}\left(\frac{1}{\alpha}-s\right)^{2}-\frac{\lambda \alpha}{2} u^{2}\right| & \leq \frac{2 \bar{b}\|Q\|_{\infty}}{\underline{b} \alpha}+\frac{\lambda \alpha}{2 \alpha^{2}}+\frac{\lambda \alpha \bar{a}^{2}}{2 \underline{b}^{2}} \leq \\
& \leq \frac{1}{\alpha}\left[\frac{\lambda}{2}+2 M\|Q\|_{\infty}\right]+\frac{\lambda \alpha \bar{a}^{2}}{2 \underline{b}^{2}} \leq \\
& \leq \delta+\delta=2 \delta .
\end{aligned}
$$

Now, from (3.19),

$$
\begin{aligned}
\int_{\Omega}\left[\delta(u-v)^{2}+\delta\right. & \left.(r-s)^{2}\right] \leq \\
& \leq\left|\int_{\Omega}(u-v)(r-s)\left(2 b s-\frac{\lambda \alpha}{2}\left(\frac{1}{\alpha}-s\right)^{2}-\frac{\lambda \alpha}{2} u^{2}\right)\right| \\
& \leq 2 \delta \int_{\Omega}|u-v||r-s|
\end{aligned}
$$

This implies $\int_{\Omega}(|u-v|-|r-s|)^{2} \leq 0$, with strict inequality if $r(x) \neq s(x)$ in a subset of $\Omega$ with positive measure. Then it follows that $r \equiv s$, and therefore $u \equiv v$ in $\Omega$.

Observe that the technique in [5] can be applied to prove uniqueness for $b=\gamma b_{0}$, where $\gamma \in \mathbb{R}$ is a sufficiently large constant and the function $b_{0}$ satisfies $[\mathbf{H}]$ (and not necessarily (3.1)). This particular case induces us to think that hypothesis (3.1) could be improved, considering the possibility of $M \geq 2$. However, the proof above strongly requires (3.1).

\section{Approximation of THE OPTIMAL CONTROL}

In this section, we consider the approximation problem to the optimal control. For it, we use the optimality system (3.8)-(3.9), because in virtue of Theorems $3.3,3.5$, the unique solution of that system describes the optimal control. We will define an iterative scheme which, under more restrictives conditions on $b$, will converge to the unique solution of (3.8)-(3.9). The main difficulty to prove this convergence is the absence of monotonicity of the terms appearing in system (3.8). Some of the ideas contained here can be found in [5, sect. 5] and [9, chap. V.].

For the sake of simplicity, we define throughout this section the functions $B, C, D: \bar{\Omega} \times \mathbb{R}^{2} \rightarrow \mathbb{R}$ as

$$
\begin{gathered}
B(x, u, p)=u\left[a-\left[b+\frac{\lambda}{2}(1-p)\right] u\right], \\
C(x, u, p)=p(a-2 b u), \\
D(x, u, p)=\frac{\lambda}{2} u(1-p)^{2} .
\end{gathered}
$$


Let us call $u_{1} \equiv w, u^{1} \equiv \frac{\bar{a}}{\underline{b}}, p_{1} \equiv 0$ and $p^{1} \equiv \frac{Q}{\underline{b}}$, where $w$ and $Q$ are defined in (3.10) and (3.3) respectively. We can find $\bar{\Gamma}>0$ such that

$$
\begin{aligned}
& B(x, u, p)+\Gamma u \text { is } \nearrow u, \nearrow p \\
& C(x, u, p)+\frac{\Gamma}{2} p \text { is } \searrow u, \nearrow p \\
& D(x, u, p)+\frac{\Gamma}{2} p \text { is } \nearrow u, \nearrow p
\end{aligned}
$$

for any $(x, u, p) \in \bar{\Omega} \times\left[0, \frac{\bar{a}}{\underline{b}}\right] \times\left[0, \frac{\|Q\|_{\infty}}{\underline{b}}\right]$ (here the symbol " $\nearrow$ " means "increasing with respect to", and " $\searrow$ " means "decreasing with respect to").

We construct inductively (observe that $u_{1}, u^{1}, p_{1}, p^{1}$ have been defined before) the sequences $\left\{u_{n}\right\},\left\{u^{n}\right\},\left\{p_{n}\right\},\left\{p^{n}\right\}$, as the unique solutions of

$$
\begin{array}{cl}
-\Delta u_{n+1}+\Gamma u_{n+1}=B\left(x, u_{n}, p_{n}\right)+\Gamma u_{n}, & \text { in } \Omega, \\
u_{n+1}=0, & \text { on } \partial \Omega, \\
-\Delta u^{n+1}+\Gamma u^{n+1}=B\left(x, u^{n}, p^{n}\right)+\Gamma u^{n}, & \text { in } \Omega, \\
u^{n+1}=0, & \text { on } \partial \Omega, \\
-\Delta p_{n+1}+\Gamma p_{n+1}=C\left(x, u^{n}, p_{n}\right)+\frac{\Gamma}{2} p_{n}+D\left(x, u_{n}, p_{n}\right)+\frac{\Gamma}{2} p_{n}, & \text { in } \Omega, \\
p_{n+1}=0, & \text { on } \partial \Omega, \\
-\Delta p^{n+1}+\Gamma p^{n+1}=C\left(x, u_{n}, p^{n}\right)+\frac{\Gamma}{2} p^{n}+D\left(x, u^{n}, p^{n}\right)+\frac{\Gamma}{2} p^{n}, & \text { in } \Omega, \\
p^{n+1}=0, & \text { on } \partial \Omega .
\end{array}
$$

We now collect the main properties of these sequences. The proof of the following assertions can be found in [5, Theorem 5.2] in a more general version.

Observe that the sequences $\left\{u_{n}\right\},\left\{u^{n}\right\},\left\{p_{n}\right\},\left\{p^{n}\right\}$, defined above satisfy 1.

and

$$
\begin{aligned}
& u_{1} \leq u_{2} \leq \ldots \leq u_{n} \leq u^{n} \leq u^{n-1} \leq \ldots \leq u^{1} \text { in } \Omega \\
& p_{1} \leq p_{2} \leq \ldots \leq p_{n} \leq p^{n} \leq p^{n-1} \leq \ldots \leq p^{1} \text { in } \Omega
\end{aligned}
$$

$$
u_{n} \nearrow u_{*}, u^{n} \searrow u^{*}, p_{n} \nearrow p_{*}, p^{n} \searrow p^{*} \text { pointwise in } \Omega \text {. }
$$

2. $u_{*}, u^{*}, p_{*}, p^{*} \in W_{0}^{2, q}(\Omega), \forall q \in(1, \infty)$. Moreover, these functions satisfy

$$
\begin{array}{cl}
-\Delta u_{*}=B\left(x, u_{*}, p_{*}\right), & \text { in } \Omega, \\
-\Delta u^{*}=B\left(x, u^{*}, p^{*}\right), & \text { in } \Omega, \\
-\Delta p_{*}=C\left(x, u^{*}, p_{*}\right)+D\left(x, u_{*}, p_{*}\right), & \text { in } \Omega, \\
-\Delta p^{*}=C\left(x, u_{*}, p^{*}\right)+D\left(x, u^{*}, p^{*}\right), & \text { in } \Omega, \\
u_{*}=u^{*}=p_{*}=p^{*}=0, & \text { on } \partial \Omega .
\end{array}
$$

with conditions

$$
w \leq u_{*}, u^{*} \leq \frac{\bar{a}}{\underline{b}} \quad ; \quad 0 \leq p_{*}, p^{*} \leq \frac{Q}{\underline{b}} .
$$

3. If $(u, p)$ is another solution of $(3.8)$ with the property

$$
u_{1} \leq u \leq u^{1}, p_{1} \leq p \leq p^{1}
$$


then

$$
u_{*} \leq u \leq u^{*}, p_{*} \leq p \leq p^{*} .
$$

Our main interest is to prove the uniqueness of solution for the system (4.1) with conditions (4.2). If it occurs, by using that $\left(u^{*}, u_{*}, p^{*}, p_{*}\right)$ is also a solution of (4.1)-(4.2), we conclude that $u_{*}=u^{*}, p_{*}=p^{*}$ in $\Omega$ and therefore $\left(u_{*}, p_{*}\right)$ is a solution of (3.8)-(3.9).

Theorem 4.1. Let us assume $[\mathbf{H}], \sigma_{1}(-a)<0$ and (3.1). Then the system (4.1)-(4.2) has a unique solution, provided that $\underline{b}$ is large enough

Proof. (Idea) To prove this theorem we use similar arguments to those given to prove Theorem 3.5. Take $\delta \equiv \sigma_{1}\left(-a+\frac{2}{M}\left(u_{1,0}-\varepsilon\right)\right)$ and $\varepsilon$ as in (3.2) and suppose $\underline{b}^{2} \geq \max \left\{b_{2}, \frac{\lambda \alpha \bar{a}^{2}}{\delta}\right\}$, where $\alpha \equiv \max \left\{\frac{2 M\|Q\|_{\infty}}{\delta}, \frac{\lambda}{\delta}\right\}$.

Let $(u, v, p, q)$ and $(U, V, P, Q)$ be two solutions of (4.1), (4.2). Equivalently $(u, v, r, s) \equiv\left(u, v, \frac{p}{\alpha}, \frac{q}{\alpha}\right)$ and $(U, V, R, S) \equiv\left(U, V, \frac{P}{\alpha}, \frac{Q}{\alpha}\right)$ are two solutions of the system

$$
\begin{array}{cc}
-\Delta u-a u+b u^{2}+\frac{\lambda}{2} u^{2}-\frac{\lambda \alpha}{2} r u^{2}=0, & \text { in } \Omega, \\
-\Delta v-a v+b v^{2}+\frac{\lambda}{2} v^{2}-\frac{\lambda \alpha}{2} s v^{2}=0, & \text { in } \Omega, \\
-\Delta r-a r+2 b v r-\frac{\lambda \alpha}{2} u\left(\frac{1}{\alpha^{2}}-\frac{2 r}{\alpha}+r^{2}\right)=0, & \text { in } \Omega, \\
-\Delta s-a s+2 b u s-\frac{\lambda \alpha}{2} v\left(\frac{1}{\alpha^{2}}-\frac{2 s}{\alpha}+s^{2}\right)=0, & \text { in } \Omega, \\
u=v=r=s=0, & \text { on } \partial \Omega .
\end{array}
$$

satisfying

$$
0 \leq r(x), s(x) \leq \frac{Q(x)}{\alpha \underline{b}}\left(\leq \frac{1}{\alpha}\right) ; w(x) \leq u(x), v(x) \leq \frac{\bar{a}}{\underline{b}}, \text { a.e. in } \Omega .
$$

From the considered hypotheses and by using similar arguments to Theorem 3.5, we deduce that $(u, v, r, s)=(U, V, R, S)$ and therefore system (4.1)-(4.2) has a unique solution.

The authors wish to thank Professor A. Cañada for his suggestions and comments in the elaboration of this paper.

\section{REFERENCES}

[1] H. Amann: Fixed points equations and nonlinear eigenvalue problems in ordered banach spaces, SIAM review, 18, 4, October 1976, 620-709.

[2] H. Berestycki and P.L. Lions: Some applications of the method of super and subsolutions, Lect. Not. Math., 782, Springer-Verlag, 1980, 16-42.

[3] J. Blat and K.J. Brown: Bifurcation of steady-state solutions in predator-prey and competition systems, Proc. of the Roy. Soc. Edinburg, 97, 1984, 21-34.

[4] A. Cañada, J.L. Gámez and J.A. Montero: An optimal control problem for a nonlinear elliptic equation arising from population dynamics, in Calculus of Variation, Applications and Computations, Longman, Pitman Research Notes in Mathematics Series, C. Bandle, J. Bemelmans, M. Chipot, J. Saint Jean Paulin and I. Shafrir, editors, 326, 1995, 35-40.

[5] A. Cañada, J.L. Gámez and J.A. Montero: Study of a nonlinear optimal control problem for diffusive Volterra-Lotka equations, to appear.

[6] D. Gilbarg and N.S. Trudinger: Elliptic Partial Differential Equations of Second Order, 2nd Edition, Springer-Verlag, Berlin, 1983. 
[7] P. Hess: Periodic-parabolic boundary value problems and positivity, Longman Group U.K. Limited, 1991.

[8] F. He, A. Leung and S. Stojanovic: Periodic optimal control for parabolic VolterraLotka type equations, Math. Methods Appl. Sci., 18, 1995, 127-146.

[9] A. Leung: Systems of nonlinear partial differential equations, The Netherlands, Kluwer Acacemic Publishers, 1989.

[10] A. Leung and S. Stojanovic: Direct methods for some distributed games, Diff. and Int. Eqns., 3, 1990, 1113-1125.

[11] A. Leung and S. Stojanovic: Optimal control for elliptic Volterra-Lotka equations, $J$. Math. Anal. Appl., 173, 1993, 603-619.

[12] L. Li and R. Logan: Positive solutions to general elliptic competition models, Diff. and Int. Eqns., 4, 1991, 817-834.

[13] S. Stojanovic: Optimal damping control and nonlinear elliptic systems, SIAMJ. Control Optim., 29, 1991, 594-608.

[14] J. Smoller: Shock waves and reaction-diffusion equations, New York, Springer, 1983. 